\title{
CONOCIMIENTOS Y ACTITUDES SOBRE VIH/SIDA DE ESTUDIANTES DE ENFERMERÍA DE LAS UNIVERSIDADES DE LA FRONTERAY AUSTRAL DE CHILE. TEMUCO - VALDIVIA. CHILE, 2004
}

\author{
KNOWLEDGE AND ATTITUDES ABOUT HIV/AIDS IN NURSING \\ STUDENTS OF TWO UNIVERSITIES OF SOUTHERN OF CHILE, 2004
}

\author{
EDITH RIVAS R* \\ ANGÉLICA RIVAS L* \\ R MAURICIO BARRÍA P ${ }^{* * *}$ \\ Catalina SePÚlVEDA $\mathrm{R}^{* * * *}$
}

\begin{abstract}
RESUMEN
Los adolescentes constituyen la población más expuesta a contraer enfermedades de transmisión sexual, entre otros aspectos, porque muchos experimentan su primera relación sexual sin protección. El VIH/SIDA persiste como una enfermedad socialmente temida y causa de discriminación, evidenciándose déficit de conocimientos sobre formas de contagio, conduciendo a actitudes perjudiciales hacia quienes padecen este mal. El objetivo del estudio fue describir los conocimientos y actitudes de estudiantes de Enfermería de las universidades de La Frontera y Austral de Chile frente al VIH/SIDA. Se ejecutó una investigación cualitativa con enfoque antropológico cognitivo para estudiar los conocimientos y actitudes de estudiantes de tercer y cuarto año de Enfermería sobre el tema en foco. Como técnica de recolección de datos se empleó la entrevista semiestructurada, analizándose la información mediante distribución de frecuencias y análisis de discursos. Se identificaron ocho dimensiones: qué es el VIH/SIDA, formas de contagio, ¿con qué se relaciona el VIH/SIDA?, prácticas de alto riesgo, medidas de prevención, ¿quiénes están expuestos al contagio?, causas de negación al examen, y propuestas de prevención. El análisis cualitativo, evidenció déficit de formación de los estudiantes respecto del manejo de pacientes y en las medidas de prevención en población de riesgo. Enfatizan la necesidad de medidas de promoción y prevención de la enfermedad. En ambos grupos se observó una tendencia biologicista en: transmisión sexual, formas de contagio, con qué se relaciona el SIDA y prácticas de alto riesgo. En medidas de prevención, se observó limitada ponderación para educación sexual y altos valores en uso del preservativo.
\end{abstract}

Palabras claves: VIH/SIDA, estudiantes de enfermería, conocimiento, actitudes.

\begin{abstract}
Adolescents make up the most exposed population to acquiring sexually transmitted diseases, among other aspects, because many experience their first sexual relation without protection. HIV/AIDS persists as a socially feared disease and cause of discrimination, showing a lack of knowledge on the forms of infection, generating detrimental attitudes towards those who suffer from the disease. The aim of the study was to describe the knowledge and attitudes of nursing students from the Universidad de la Frontera and the Universidad Austral de Chile, in relation to HIV/AIDS. Qualitative research with anthropologic cognitive approach was executed, to study the knowledge and attitudes of nursing students of third and fourth year about the topic in focus. For data collection, the semi structured interview was used, analyzing the information by frequency distribution, and discourse analysis. Eight dimensions were identified: What is HIV/AIDS? ; Ways of infection; What is related to the HIV/AIDS? ; High risk practices; Prevention methods; Who are exposed to infection?; Causes of refusal of HIV/AIDS tests; and Proposal of prevention. The qualitative analysis, showed an educational deficit about the care of patients and prevention measures to the risk population. The students emphasize the need of promotion and prevention measures for the disease. In both groups, a biologicist trend was observed in: Sexual transmission, Ways of infection, What is related to the HIV/AIDS? and High risk practices. In prevention methods, a limited percentage was given to sexual education and the highest to the use of condoms.
\end{abstract}

Keywords: AIDS/HIV, nursing students, knowledge, attitudes.

Fecha recepción: 02/04/08 Fecha aceptación: 02/04/09

*Enfermera, Doctora (c) en Enfermería, docente Universidad de la Frontera, Temuco. E-mail: erivas@ufro.cl

${ }^{* *}$ Enfermera, UCI Neonatal, Hospital Hernán Henríquez Aravena, Temuco. E-mail: ayrivasl@gmail.com

${ }^{* * *}$ Enfermero, M.Sc. Epidemiología Clínica, Universidad Austral de Chile, Valdivia. E-mail: rbarria@uach.cl

${ }^{* * * *}$ Enfermera, Clínica Alemana de Temuco. E-mail: catasepril@gmail.com 


\section{INTRODUCCIÓN}

En el contexto epidemiológico, el VIH/SIDA es una enfermedad temida que no discrimina estratos sociales, económicos ni culturales. A la fecha, más de 70 millones de personas se han infectado de SIDA, siendo la $4^{\text {a }}$ causa de muerte a nivel mundial. Los más altos índices de personas infectadas se encuentran en la población adulta joven (un tercio de las personas entre 15 y 24 años de edad) y se estima que la gran mayoría no sabe que vive con VIH (1).

En la actualidad, más de $95 \%$ de las personas con VIH/SIDA residen en países en vías de desarrollo, concentrándose $71,2 \%$ en África Subsahariana. En América Latina y el Caribe, más de 2 millones de personas viven con VIH/SIDA, incluidas las 200.000 que adquirieron la infección el año 2003. Alrededor de 100.000 personas murieron de SIDA en el mismo periodo (índice más alto de víctimas mortales, después de África y Asia) (2).

En Chile, el primer caso de SIDA fue notificado en 1984 y hasta el año 2001 existían 4.749 individuos con SIDA, 5.276 con VIH y 3.230 fallecidos. La vía sexual persiste como el principal mecanismo de exposición, siendo responsable de $94 \%$ de los casos en 2001. El grupo más afectado se encuentra entre los 20 y 49 años, especialmente hombres, existiendo una tendencia a la heterosexualización y feminización de la epidemia.

En la Región de la Araucanía, entre 1989 y abril de 2005 se notificaron 305 personas con VIH/SIDA. Al comparar las incidencias acumuladas regionales, al 2003 se observó un ascenso tanto de la IX Región de la Araucanía, desde el lugar $11^{\circ} \mathrm{al} 8^{\circ}$, como de la X región de Los Lagos, desde el 10 al 9o (3). Desde 1993 a octubre de 2005 se han registrado 164 casos de infección y 36 fallecidos en la comuna de Temuco.

Una de las poblaciones más vulnerables continúa siendo la adolescente, debido a que constituye la etapa del desarrollo en la que se producen transformaciones significativas del desarrollo biológico y psicológico. Para Schutt-Aine y Maddaleno (4), "la adolescencia comprende transformaciones significativas del desarrollo biológico del cuerpo, asociadas a la madurez sexual y psicológica. Cambios en el pensamiento, capacidad de abstracción y una importante motivación por diferenciarse e individualizarse de los modelos paternos". Es en esta etapa donde una proporción importante de los jóvenes experimenta su primera relación sexual sin protección, exponiéndose a infecciones de transmisión sexual, VIH/SIDA y embarazos no deseados. Por su importancia en el curso de la vida, existe un debate sobre los programas de educación sexual y de prevención dirigidos a este grupo etario, a partir de las políticas estadounidenses, basadas exclusivamente en el fomento de la abstinencia (5).

En nuestro país, en los últimos años, se han desarrollado políticas de educación sexual y estrategias que fomentan una sexualidad responsable, estableciendo consejerías en los Servicios de Salud y establecimientos educacionales. Desde 1970 a 1973 se dio inicio a la Institucionalización de la Educación Sexual y desde 1995 se han desarrollado estudios en atención integral adolescente, como la publicación "Hacia una política de educación sexual para el mejoramiento de la calidad de la educación" (6).

\section{Estigma, conocimientos, actitudes}

El SIDA continúa siendo afectado por la estigmatización al igual que las patologías mentales y la discapacidad. Se asocia con estereotipos sociales, positivos o negativos (7). El estigma se refiere a "signos corporales con los cuales se intentaba exhibir algo malo y poco habitual en el estatus moral de quien los presentaba" (8). Actualmente, permanece, es desacreditador y está basado en factores como: edad, casta, clase, color, etnia, religión, sexo, orientación sexual, entre otros $(7,8)$. Al estigmatizar a un individuo "dejamos de verlo como una per- 
sona total y corriente para reducirlo a un ser aficionado y menospreciado" (8).

El estigma da paso a la discriminación, tomando los individuos actitudes activas o pasivas perjudiciales frente a las personas que padecen este mal. La Convención Interamericana sobre la eliminación de todas las formas de discriminación contra las personas con discapacidades, la describe como cualquier diferencia, exclusión o restricción que viole los derechos humanos y las libertades de las personas (7).

Estudios sobre el VIH/SIDA realizados en estudiantes universitarios de enfermería, arte, matemáticas y medicina, han revelado diferencias respecto de la percepción que presentan frente a los pacientes con SIDA. En EEUU, Katz et al. (9) destacan el rechazo y negativa evaluación por parte de los estudiantes, definiéndolos responsables de su propia enfermedad; indican no sentir miedo al contagio, sino aversión al comportamiento sexual. Por su parte, Snowden (10) señala que menos del 5\% tiene actitudes negativas hacia el usuario infectado, cifra menor en estudiantes de enfermería. El estudio concluye que se deben realizar modificaciones en la formación de los alumnos, pues éstos refieren que no existen instancias de discusión y si existieran causarían gran impacto.

En VIH/SIDA la clave es la prevención, educar en la solidaridad de la humanidad, practicar el principio de corresponsabilidad hacia todos los seres de la Tierra; reconociendo su dignidad y necesidad de respeto. Se apuesta por una concepción de las relaciones interpersonales, donde el respeto recíproco sea reflejo de la virtud de la castidad (11), donde la autonomía y la interdependencia se interpreten siempre vinculadas (12), contribución a la humanización de la sexualidad como dimensión humana esencial.

Los trabajadores de la salud tienen la responsabilidad y el compromiso de dar a todos los usuarios atención de buena calidad, sin importar su raza, religión, sexo, orientación sexual, diagnóstico y/o pronóstico. Sin embargo, incluso en los servicios de salud, donde las personas que viven con VIH/SIDA buscan y esperan recibir atención y cuidados, comúnmente enfrentan problemas de estigma y discriminación (7).

Se ha recomendado introducir en la praxis de la educación las modificaciones necesarias que promuevan la generación de sensibilidad y conciencia de responsabilidad, promover en la escuela y familias educación en valores en el ámbito de las relaciones, los afectos y la sexualidad. Crear instancias de conversación y foros referentes al SIDA y sus implicancias, generando una cultura en sintonía con los valores del respeto e interés por el más débil.

Por otra parte, desde 1991, el Ministerio de Salud de Chile ha desarrollado 5 campañas nacionales de prevención del VIH/SIDA, con el objetivo de que la población adopte conductas preventivas.

En Chile, las enfermeras han reiterado la necesidad de explorar en los patrones sexuales, como una función trascendental, para indagar en problemas de salud, situaciones y conflictos ocasionados por los nuevos estilos de vida. Por esto, se han considerado como sujetos de estudio a jóvenes estudiantes, por constituir una población que a raíz de su etapa de desarrollo tanto fisiológica como sicológica presentan un alto grado de exposición al riesgo de transmisión y donde es necesaria la información respecto de factores protectores para la toma de decisiones en conducta sexual.

El conocimiento de VIH/SIDA es limitado en estudiantes de la educación superior, y se considera relevante aportar al estudio empírico sobre el tema. Por tal motivo se realizó una profundización de la temática de conocimiento y actitudes en alumnos de ambos sexos, en las carreras de Enfermería de dos universidades del sur de Chile, profesión que por su naturaleza, de tratar con las personas en situaciones de enfermedad y salud, tiene un imperativo ético valórico. Socialmente, los alumnos de enfermería deben cumplir un rol de joven, donde las presiones 
sociales son variadas y de difícil manejo y donde el autocuidado es la herramienta de mayor fuerza. Pero además, deben cumplir un rol de futuro profesional responsable de la gestión del cuidado, elemento hoy más que nunca vigente y determinado en el área de la promoción de la salud. Es en esta etapa donde se puede intervenir para modificar la estigmatización de las personas afectadas con esta enfermedad, teniendo una mirada acogedora y de respeto por quien necesita de nuestros cuidados.

\section{MATERIAL Y MÉTODO}

Investigación cualitativa con enfoque antropológico cognitivo, que estudia cómo los sujetos de diferentes culturas adquieren información y la procesan para generar decisiones y actuar de acuerdo con los valores aceptados como válidos y normas culturales (13). El objetivo del estudio fue describir los conocimientos y actitudes frente al VIH/SIDA, de estudiantes de Enfermería de las universidades de La Frontera y Austral de Chile, con el propósito de contribuir al mejoramiento de la formación profesional de enfermeras/os, en materia de prevención de conductas de riesgo en VIH/SIDA.

Los sujetos de estudio fueron 78 alumnos de $3^{\circ}$ y $4^{\circ}$ año de Enfermería de la Universidad de la Frontera (UFRO) y Universidad Austral de Chile (UACh) durante el año 2003. En una primera fase se aplicó la técnica de respuesta libre para explorar los términos que expresan los alumnos cuando se evalúa los conocimientos y actitudes respecto de VIH/ SIDA. La información fue recogida a través de una entrevista semiestructurada, apoyada por preguntas generadoras de diálogo como: ¿qué es para ti el SIDA?, formas de contagio, ¿con qué se relaciona el VIH/SIDA?, entre otras, extraídas de la investigación de Caballero y Uribe (14).

Se realizó análisis cuantitativo con deter- minación de frecuencias y análisis cualitativo mediante semántica estructural, utilizando el principio de saturación, que permitió identificar dimensiones teóricas y categorías analíticas.

Para el estudio se obtuvo consentimiento informado para la participación, grabación y transcripción de entrevistas. Las entrevistas se realizaron en dependencias de ambas universidades que ofrecían la estructura apropiada para resguardar la privacidad.

\section{RESULTADOS Y DISCUSION}

La edad promedio de los estudiantes de la UFRO y UACh fue 23,5 años $( \pm 1,2)$ y 22,4 años $( \pm 3,8)$, respectivamente. La distribución por sexo fue similar, encontrándose un claro predominio de mujeres tanto en la UFRO $(91,9 \%)$ como en la UACh $(95,3 \%)$.

En el análisis se identificaron las dimensiones: Qué es el VIH/SIDA, formas de contagio, con qué se relaciona VIH/SIDA, prácticas de alto riesgo, medidas de prevención, quiénes están expuestos al contagio, causas de negación al examen de detección y propuestas de prevención. Los totales corresponden a la suma de las categorías analíticas de cada dimensión (tablas 1, 2 y 3).

Qué es el VIH/SIDA: Los estudiantes de la UFRO consideraron principalmente que el VIH/SIDA es una enfermedad de transmisión sexual $(31,8 \%)$, en tanto los de la UACh, que se trata de una enfermedad del sistema inmune $(29,8 \%)$. En contraste, las categorías enfermedad con desgaste psicológico y pandemia, fueron los aspectos menos reconocidos por los estudiantes de la UFRO y UACh, respectivamente (Tabla 1A).

Formas de contagio: En ambas casas de estudio, el mayor porcentaje de estudiantes atribuyó como principales formas de contagio a la categoría transmisión sexual (UFRO 26,8\%; UACh $38,1 \%$ ) y transfusiones (UFRO 22,8\%; UACh $26,1 \%$ ). Vale destacar que un porcen- 
taje no despreciable de estudiantes reconoció como forma de contagio el uso inadecuado de material corto punzante (UFRO 7,1\%; UACh 9,8\%) (Tabla 1B).

Con qué se relaciona el VIH/SIDA: En este aspecto, los estudiantes de la UFRO consideraron como principales condiciones que se relacionan con el VIH/SIDA la homosexualidad y promiscuidad (21,3\%), similar a lo encontrado en los estudiantes de la UACh en que estas categorías alcanzaron 20,7 y 22,4\% respectivamente (Tabla $1 \mathrm{C}$ ).

Prácticas de alto riesgo: Consecuentemente con la categoría anterior, el mayor porcentaje de estudiantes de la UFRO atribuyó como principales prácticas de alto riesgo a la homosexualidad y la promiscuidad (21,3\%). Por su parte los alumnos de la UACh valoraron como principal práctica de riesgo el sexo sin protección, concentrando esta opción $26,2 \%$ (Tabla 2A).

Medidas de prevención: Se reconoce en los estudiantes de ambas universidades que la abstinencia sexual es la principal medida preventiva, alcanzando $50 \%$ en estudiantes de la UACh y $22,3 \%$ en los de la UFRO. Estos últimos valoraron como igualmente importante el factor pareja única (Tabla 2B).

Quiénes están expuestos a contagio: La distribución en este aspecto fue más heterogénea destacando las categorías "trabajadoras de salud" (UFRO 29,8\%; UACh 21,4\%) y los grupos de riesgo (UFRO 21\%; UACh 29,4\%) (Tabla 2C).

Causas de negación al examen de detección: La mitad de los estudiantes de ambas universidades identificaron el miedo como principal causa de negación al examen de detección (Tabla 3A).

Propuestas de prevención: Como era de esperar, las propuestas de prevención se centraron en el uso de preservativo en los estudiantes de la UFRO y pareja única, estable y fidelidad en los de la UACh. Llama la atención que la categoría información fue mínimamente considerada (Tabla 3B).

En la segunda fase se analizaron los dis- cursos de los alumnos, utilizando el método de semántica estructural y se identificaron las dimensiones político-sociales cuyas categorías fueron: necesidad de dar respuestas, limitado acceso a información, inexistencia de resolución de problemas y déficit de prevención; la dimensión biologicista con las categorías: grupos vulnerables, riesgos, existencia de evidencia y la dimensión valórico-conductual y sus categorías comportamientos sexuales, actitudes dolorosas (rabia, dolor, miedos, culpa), actitud de déficit de formación y mitos (Tabla 4).

En conocimientos se rescatan textos alusivos al sentir de los alumnos frente a una situación de ayuda: "No estoy preparada para el acompañar al paciente en el proceso"; "No cuento con herramientas para establecer una relación de confianza con el paciente"; ; como establecer una relación de ayuda?; "tengo dificultades para tratar y cuidarlos"; "tengo temor al contagio". De los textos se desprenden dos ideas, una respecto a las falencias en la formación de asistencia o ayuda y la segunda el temor al contagio.

En materia de prevención, los estudiantes enunciaron: "Son medidas sin impacto y Enfermería debería tener mayor participación". Otro texto se refiere al déficit de trabajo en población escolar de las enfermeras: "No se trabaja con los escolares y adolescentes, las universidades deberían preocuparse de la situación y especialmente Enfermería".

Otro discurso alude a la participación: "Enfermería debería tener mayor visibilidad dentro del enfoque multiprofesional, con programas continuos y un enfoque de cuidado humano, esto como política".

En actitudes, los textos muestran sentimientos de compartir el dolor de los pacientes, pero también existe un sentir respecto de la participación: "Me da mucha rabia e impotencia lo que pasa con los pacientes con Sida, a nadie le interesan me da mucho dolor"; "siento que hay mucha discriminación, es como un estigma, hay mucha falta de apoyo". 
Tabla 1. Distribución porcentual de estudiantes por universidad y categorías y subcategorías de respuesta sobre las características de la enfermedad.

\begin{tabular}{|c|c|c|}
\hline \multirow{2}{*}{ Categoría/subcategorías } & \multicolumn{2}{|c|}{ Universidad } \\
\hline & UFRO (\%) & UACh (\%) \\
\hline \multicolumn{3}{|l|}{ A. Qué es el VIH/SIDA } \\
\hline Enfermedad de transmisión sexual & 31,8 & 26,4 \\
\hline Enfermedad mortal & 25,2 & 14,0 \\
\hline Pandemia & 8,4 & 1,7 \\
\hline Enfermedad infecto contagiosa & 13,6 & 21,0 \\
\hline $\begin{array}{l}\text { Enfermedad del Sistema inmune } \\
\text { Enfermedad con desgaste psicológico }\end{array}$ & $\begin{array}{c}14,7 \\
6,3\end{array}$ & $\begin{array}{c}29,8 \\
7,0\end{array}$ \\
\hline \multicolumn{3}{|l|}{ B. Formas de contagio. } \\
\hline Por transmisión sexual & 26,8 & 38,1 \\
\hline Por transmisión por fluidos corporales & 15,0 & 6,5 \\
\hline Por transfusiones & 22,8 & 26,1 \\
\hline Por uso de drogas & 11,0 & 1,1 \\
\hline Transmisión madre-hijo & 15,0 & 17,4 \\
\hline Lactancia materna & 2,4 & 1,1 \\
\hline Uso inadecuado material corto punzante & 7,1 & 9,8 \\
\hline \multicolumn{3}{|l|}{ C. Con qué se relaciona el VIH/SIDA } \\
\hline Hábitos sexualidad insegura & 10,1 & 6,9 \\
\hline Drogadicción & 18,0 & 12,1 \\
\hline Homosexualidad & 21,3 & 20,7 \\
\hline Prostitución & 9,0 & 3,4 \\
\hline Conductas de riesgo & 5,6 & 3,9 \\
\hline $\begin{array}{l}\text { Promiscuidad } \\
\text { Uso de drogas endovenosas }\end{array}$ & $\begin{array}{c}21,3 \\
4,5\end{array}$ & $\begin{array}{l}22,4 \\
14,1\end{array}$ \\
\hline Con pareja no única & 10,2 & 16,6 \\
\hline
\end{tabular}

UFRO: Universidad de La Frontera; UACh: Universidad Austral de Chile. 
Tabla 2. Distribución porcentual de estudiantes por universidad y categorías y subcategorías de respuesta sobre aspectos relacionados con el contagio.

\begin{tabular}{|l|c|c|}
\hline \multirow{2}{*}{ Categoría/subcategorías } & \multicolumn{2}{|c|}{ Universidad } \\
\cline { 2 - 3 } & UFRO (\%) & UACh (\%) \\
\hline A. Prácticas de alto riesgo & 10,1 & 26,2 \\
\hline Sexo sin protección & 18,0 & 7,0 \\
Drogadicción & 21,3 & 20,4 \\
Homosexualidad & 9,0 & 13,1 \\
Prostitución & 5,6 & 10,7 \\
Conductas de riesgo & 21,3 & 5,1 \\
Promiscuidad & 4,5 & 7,1 \\
Uso de drogas endovenosas & 10,2 & 10,4 \\
Pareja no única & \multicolumn{2}{|c|}{} \\
\hline B. Medidas de prevención & 22,3 & 5,9 \\
\hline Pareja única & 21,2 & 29,7 \\
Uso preservativo & 22,3 & 50,0 \\
Abstinencia sexual & 7,0 & 8,5 \\
Educación sexual & 11,8 & 4,2 \\
Conducta sexual segura & 15,3 & 1,7 \\
Precaución fluidos corporales & \multicolumn{2}{|c|}{} \\
\hline C. Quienes están expuestos al contagio & 12,3 & 13,2 \\
\hline Hijo de madre VIH & 29,8 & 21,4 \\
Trabajadoras de salud & 21,0 & 29,4 \\
Grupos de riesgo & 26,3 & 15,3 \\
Personas sin precaución & 10,5 & 20,6 \\
Drogadictos & \multicolumn{2}{|c|}{} \\
\hline UrO: Univessidad La & \\
\hline
\end{tabular}

UFRO: Universidad de La Frontera; UACh: Universidad Austral de Chile. 
Tabla 3. Distribución porcentual de estudiantes por universidad y categorías y subcategorías de respuesta sobre aspectos relacionados con diagnóstico y prevención.

\begin{tabular}{|l|c|c|}
\hline \multirow{2}{*}{ Categoría/subcategorías } & \multicolumn{2}{|c|}{ Universidad } \\
\cline { 2 - 3 } & UFRO (\%) & UACh (\%) \\
\hline \multirow{2}{*}{$\begin{array}{l}\mid \\
\text { A. Por qué las personas se niegan a realizarse el } \\
\text { examen de detección }\end{array}$} \\
\hline Miedo & 49,2 & 50,5 \\
Falta educación & 18,0 & 12,5 \\
Desconocimiento & 18,0 & 22,5 \\
Por sanción social & 11,5 & 10,2 \\
Diagnóstico mortal & 3,3 & 4,3 \\
\hline B. Propuestas de prevención & \multicolumn{2}{|l}{} \\
\hline Responsabilidad & 11,5 & 26,5 \\
Información & 7,7 & 4,7 \\
Uso preservativo & 38,5 & 17,2 \\
Abstinencia sexual & 5,8 & 6,2 \\
Pareja única, estable y fidelidad & 19,2 & 29,7 \\
Educación sexual colegios & 17,3 & 15,6 \\
\hline
\end{tabular}

UFRO: Universidad de La Frontera; UACh: Universidad Austral de Chile.

Tabla 4. Dimensiones teóricas y categorías analíticas

\begin{tabular}{|c|c|}
\hline Dimensiones teóricas & Categorías analíticas \\
\hline | Político social & $\begin{array}{l}\text { 1. Necesidad de dar respuestas } \\
\text { 2. Limitado acceso a información } \\
\text { 3. Inexistencia de resolución al problema } \\
\text { 4. Déficit de prevención }\end{array}$ \\
\hline || Biologicista & $\begin{array}{l}\text { 1. Grupos vulnerables } \\
\text { 2. Riesgos } \\
\text { 3. Existencia de evidencia }\end{array}$ \\
\hline III Valórico- conductual & $\begin{array}{l}\text { 1. Comportamientos sexuales } \\
\text { 2. Actitudes dolorosas: rabia, dolor, miedos, culpa } \\
\text { 3. Déficit de formación y mitos }\end{array}$ \\
\hline
\end{tabular}


Los hallazgos encontrados en los estudiantes de ambas universidades son similares, observándose dimensiones eminentemente biologicistas en las categorías: transmisión sexual, formas de contagio, con qué se relaciona el VIH/SIDA y prácticas de alto riesgo.

En medidas de prevención, llama la atención el ínfimo porcentaje que ambos grupos le atribuyen a la educación sexual y conducta sexual segura, y los altos porcentajes atribuidos al uso del preservativo.

Respecto de quienes están expuestos a infectarse, los mayores porcentajes corresponden a grupos de riesgo (personas sin precaución, drogadictos y trabajadoras de la salud). El hijo de madre VIH/SIDA es considerado de bajo riesgo en ambas universidades y en relación a la dimensión "quienes se niegan a realizarse el examen", los mayores porcentajes se asocian a personas que presentan miedo, desconocimiento y falta de educación. Al respecto, un estudio reporta que "no puede negarse que las características de la enfermedad generan creencias, miedos y temores sobre el contagio, temor al que no está ajeno el trabajador de salud. Los mecanismos por los cuales se puede adquirir la enfermedad, principalmente en el caso de usuarios de drogas, homosexuales y trabajadoras sexuales, conlleva a prejuicios sociales que culpabilizan al usuario" (15).

En la dimensión "propuestas para no infectarse", los alumnos señalan el uso del preservativo y responsabilidad como los más importantes.

En el análisis de los discursos, los alumnos manifiestan presentar sentimientos y actitudes dolorosas frente al paciente con VIH/SIDA como rabia, dolor, miedos, culpa, actitud de déficit de formación y mitos, la relación con el problema de la estigmatización es recurrente en los textos. Similarmente, Varas et al. (16) confirman este sentimiento, aduciendo que la "estigmatización y discriminación produce grandes cambios en el área emocional, pues los afectados sienten que no son aceptados en la sociedad".

En conocimientos se rescatan textos alu- sivos al sentir de los alumnos frente a una situación de ayuda, "No estoy preparada para el acompañar al paciente en el proceso". Al respecto Bartlett y Finkbeiner (17) refieren que en la transmisión de la información surgen miedos como por ejemplo, ¿cómo decirles?, ¿cómo manejar sus inquietudes?

En otro texto de materia de prevención enuncian "Enfermería debería tener mayor participación" y "no se trabaja con los escolares y adolescentes," idea reforzada por el trabajo de Garwick et al (18), quienes señalan que la relación de apoyo con un cuidador puede tener efectos positivos sobre los comportamientos sexuales de las adolescentes.

Conjuntamente, se ha visto que las actitudes de los trabajadores de la Salud hacia quienes padecen de VIH/SIDA influyen de manera decisiva en la forma en que las personas y las instituciones sociales responden frente a esta enfermedad (19).

Otro discurso se refiere a la participación expresando que "enfermería debería tener mayor visibilidad dentro del enfoque multiprofesional, con programas continuos y un enfoque de cuidado humano". En ese contexto, se ha desatacado que el objetivo de la Atención de Enfermería es brindar apoyo y contribuir a lograr una mejor calidad de vida del paciente, incrementar la autocomprensión y autoconocimiento, mejorar la comunicación, promover hábitos de vida saludable, preventiva y de autocuidado (20).

Un estudio de claves físicas y psicológicas de cuidado de enfermería para pacientes con VIH, menciona que la enfermería es holística, por tanto considera la totalidad del individuo que vive con el virus de inmunodeficiencia humano: cuerpo, mente y espíritu.

Similarmente, Caetano et al. señalan que es importante usar la teoría de autocuidado de Orem, permitiendo a individuos percibir sus aspectos orgánicos, físicos, afectivos, sociales, culturales y espirituales; haciendo a las enfermeras identificarse en la capacidad del autocuidado (21). En esta misma línea, se ha reconocido como importante 
evaluar la demanda de cambio percibida, el autocuidado y el grado de dificultad de cuidado usando la Escala de Carga de autocuidado (22).

Conjuntamente, Da Costa et al. (23) reiteran que las expectativas de los pacientes son: más cuidado humano, más atención, contacto verbal, más afectividad del equipo enfermero, una atención calificada y digna. Además, deberían ser informados diariamente sobre las vulnerabilidades a que ellos son sometidos durante la hospitalización.

En actitudes los textos muestran sentimientos de compartir el dolor de los pacientes, pero también hay un sentimiento de participación: "Me da mucha rabia e impotencia lo que pasa con los pacientes con Sida, a nadie le interesan... me da mucho dolor"; "siento que hay mucha discriminación, es como un estigma, hay mucha falta de apoyo".

Al respecto, Luco (24) se refiere a las alteraciones fisiopatológicas y cambios en la imagen corporal que provocan desequilibrios en el terreno emocional. En este sentido consideramos que si bien ha mejorado la notificación y consejería, persisten los mitos $\mathrm{y}$ actitudes negativas.

En educación en VIH/SIDA se deben generar diseños centrados en conductas de riesgo específicas, usar modelos teóricos reconocidos, métodos pedagógicos interactivos, clarificación de valores, resistencia a la presión social y entrenamiento en habilidades de comunicación, de esta forma se dará respuesta a la gestión del cuidado (24).

El análisis cualitativo arrojó textos alusivos al déficit de formación en el manejo de pacientes, miedo, inseguridad y falta de medidas de prevención que produzcan impacto en la población en riesgo. Se ha señalado que, de existir mayor énfasis en las medidas de promoción de la salud y prevención de la enfermedad, disminuiría la discriminación hacia las personas que padecen VIH/SIDA.

\section{CONCLUSION}

La investigación arrojó hallazgos centrados eminentemente en el aspecto físico-biológico, reflejando vacíos de conocimientos en Salud Mental, déficit en atención integral y manejo de redes de apoyo. A nuestro juicio se deben reestructurar los planes de estudio y reforzar los contenidos de Salud Mental, desarrollando una línea de trabajo abierta con organizaciones de cuidado de VIH/SIDA. Las enfermeras/os deben actualizar y reflexionar, por principio, en forma permanente para generar una educación abierta, dinámica, plural, autogestiva, crítica, competente y creativa, que capacite al futuro profesional para incursionar de forma eficiente en el escenario que la práctica global está demandando.

\section{REFERENCIAS}

1. Caetano JA, Pagliuca LM. Self-care and HIV/AIDS patients: nursing care systematization. Rev Lat Am Enfermagem. 2006 May;14(3):336-45.

2. Programa Conjunto de las Naciones Unidas sobre VIH/SIDA. Situación de la Epidemia del SIDA. Ginebra: Mediterráneo; 2003.

3. Comisión Nacional del SIDA. Boletín Epidemiológico VIH/SIDA. Boletín Epidemiológico semestral CONASIDA No 15. 2003, disponible en: http://www.minsal.cl/ ici/conasida/documentos/boletin_15.pdf . [Extraído el 29 de diciembre, 2009].

4. Schutt-Aine, Maddaleno M. Salud sexual y desarrollo de adolescentes y jóvenes en las Américas. Implicaciones en programa y políticas. Washington, DC: Organización Panamericana de la Salud; 2003.

5. Lindberg LD, Santelli JS, Singh S. Changes in formal sex education: 19952002. Perspect Sex Reprod Health. 2006 Dec;38(4):182-9. 
6. Olavarría J. La política de Educación Sexual del Ministerio de Educación de Chile. Consideraciones para una evaluación. Seminario Internacional Equidad de Género en las Reformas Educativas de América Latina; 2005.

7. Foreman M, Lyra P, Breinbauer C. Comprensión y respuesta al estigma y a la discriminación por el VIH/SIDA en el sector salud. Washington, DC: Organización Panamericana de la Salud; 2003.

8. Goffman E. Estigma, la identidad deteriorada. $3^{a}$ edición. Buenos Aires: Amorrortu;1986.

9. Katz I, Hass RG, Parisi N, Astone J, McEvaddy D, Lucido DJ. Lay people's and health care personnel's perceptions of cancer, AIDS, cardiac, and diabetic patients. Psychol Rep 1987 Apr;60(2):615-29.

10. Snowden L. An investigation into whether nursing student alter their attitudes and knowledge levels regarding HIV infection and AIDS following a 3-year programme leading to registration as a qualified nurse. J Adv Nurs 1997 Jun;25(6):1167-74.

11. Thévenot X. Pautas éticas para un mundo nuevo. Navarra: Verbo Divino. 1988.

12. Camps V.Virtudes públicas. Madrid: Editorial Espasa Calpe.1990.

13. Bernard H. Research Methods in Cultural Anthropology. $4^{a}$ edición. Altamira. 1995.

14. Caballero R, Uribe P. Exploración de significados culturales sobre el Sida en adolescentes de Guadalajara. Investigación Cualitativa en Salud. Perspectivas desde el Occidente de México. $1^{a}$ edición. Universidad de Guadalajara. Serie Medicina Social; 1998.

15. Herrera A, Cianelli R, Ferrer L. Estigma y discriminación a usuarios con VIH/SIDA, por parte de los trabajadores de la Salud. Horiz Enferm. 2005; 16:49-58.
16. Varas N, Serrano I, Toro J. Stigma and social difference: HIV/AIDS. San Juan: Ed. Huracán; 2004.

17. Bartlett J, Finkbeiner A. Guía para vivir con VIH y SIDA; desarrollada en la Clínica de SIDA de Johns Hopkins. México: Ed. Diana. 1996.

18. Garwick A, Nerdahl P, Banken R, Muenzenberger-Bretl L, Sieving R. Risk and protective factors for sexual risk taking among adolescents involved in Prime Time. J Pediatr Nurs. 2004 Oct; 19(5):340-50.

19. Vidal F. Factores que influyen en las actitudes públicas hacia el SIDA. [Tesis de Magíster]. Instituto de Sociología, Universidad Católica de Chile, Santiago; 1995

20. Sepúlveda C, Afani A. AIDS. Santiago: Mediterráneo. 1994

21. O'Brien ME, Pheifer WG. Physical and psychosocial nursing care for patients with HIV infection. Nurs Clin North Am. 1993 Jun; 28(2):303-16.

22. Oberst MT, Hughes SH, Chang AS, McCubbin MA. Self-care burden, stress appraisal, and mood among persons receiving radiotherapy. Cancer Nurs. 1991 Apr;14(2):71-8.

23. Da Costa JP, Da Silva LM, Da Silva MR, Miranda KC. Expectativas de pacientes com HIV/AIDS hospitalizados, quanto à assistência de enfermagem. Rev Bras Enferm. 2006 Mar;59(2):172-6.

24. Luco A. Prevención e intervención psicológica en pacientes con SIDA. Rev Chil Psicol. 1990;2(11):17-23.

25. Anastasio C, McMahan T, Daniels A, Nicholas PK, Paul-Simon A. Self-care burden in women with human immunodeficiency virus. J Assoc Nurses AIDS Care. 1995 May;6(3):31-42. 
